\title{
Cavity length and re-entrant jet in 2-D sheet cavitation
}

\author{
I. Castellani \\ Department of Mechanical and Structural Engineering, \\ University of Trento, Italy.
}

\begin{abstract}
Some features of hydrodynamic cavitation have been investigated in this experimental study to understand the role of the re-entrant jet in the formation of sheet (or cloud) 2-D cavitation. Velocity profiles, frequency contents of the cavity, pressure coefficients and pressure pulses spectrum have been measured in isothermal conditions. Physical quantities of interest were found to be greatly affected by the rate of pressure increase. Flow separation, surge instability, hysteresis, rebounds, splashes, choking and re-entrant jets have been observed. A correlation has been found between the cavitation number and the cavity length. The frequency content was detected by a new method that produces a spectrum at high Strouhal numbers. A large band without a peak was observed for the highest frequencies, e.g. greater than $1000 \mathrm{~Hz}$. The role of the re-entrant jet in breaking the cavity was not found to be as much important as it is currently believed. The present results, obtained through thousands of experiments, may be of interest to predict cavity formation. Keywords: cavitation, re-entrant jet, frequency content, laser measurements, pressure pulses, wall effects, choke, hysteresis, rebound, splash.
\end{abstract}

\section{Introduction}

The present work originates from previous literature results about sheet-cloud 2-D cavitation, e.g. Astolfi et al. [8], Franc and Lauterborn [9], Lindau [12] and Sato and Shimojo [14], who discussed features such as periodicity, re-entrant jet, pressure gradient, rebound, wall effect and morfology.

The experimental apparatus is described at length in Castellani [15, 16]. It comprises a high speed water tunnel, a centrifugal pump $(120 \mathrm{l} / \mathrm{min}$ and $25 \mathrm{~m}$ maximum head) and a vacuum pump. The temperature was always kept constant at 
$20^{\circ} \mathrm{C}$ and the pressure could rise up to $7 \mathrm{bar}$. The test chamber is a perspex Venturi channel, where a perspex hydrofoil is allocated on the floor, at the minimum area section (throat) of the chamber. The $x$-axis is horizontal and oriented streamwise; the $y$-axis is vertical, upward; the $z$-axis follows the left-handed cartesian rule. The channel width (in $z$ direction) is $80 \mathrm{~mm}$, while the throat height is $9.8 \mathrm{~mm}$. The undisturbed flow reference station, labelled " 0 ", has been chosen for computing velocity, static pressure, cavitation index and pressure coefficient. The chord length of the hydrofoil is $c=44.42 \mathrm{~mm}$ at a fixed angle of attack $\alpha=5^{\circ}$. The hydrofoil has a spanwise groove, located at the throat section, which helps in fixing exactly the starting point of the cavity. The volumetric flow rate $Q$, the static pressure $p_{0}$ and the temperature $T$ could be independently controlled.

The relevant nondimensional parameters are:

(i) the cavitation index $\sigma_{0}$, where $\sigma_{0 i}$ stands for the inception index;

(ii) the pressure coefficient $C_{p}$;

(iii) the Strouhal number $S t$, based on the double of the cavity length $2 L$.

All of the experiments were performed under steady state conditions. The cavitation stages were always obtained by decreasing the pressure at given velocities. The velocity fields was measured by the LDA technique. The wall effect was taken into account performing spanwise measurements: a distance from the wall $z=5 \mathrm{~mm}$ was found to be sufficient to overcome the boundary layer influence.

At a given $V_{0}$, the static pressure $p_{0}$ was reduced until the cavitation inception $\left(\sigma_{0 i}\right)$ was reached, then it was further reduced step by step, recording the cavitation index for each condition, until $L / c \simeq 0.66$. The pressure was then increased until the cavitation disappeared. This cycle was repeated five times at any tested velocity to detect a possible hysteresis.

The frequency measurements of cavity were performed in three ways:

(i) by a high speed video camera of the scattered (indirect) or direct laser light;

(ii) by a mean speed acquisition of the whole cavity;

(iii) by a piezoresistive pressure transducer $(180 \mathrm{kHz}$ response and $140 \mathrm{bar})$, allocated into the hydrofoil.

The acquisition interval is about $5.5 \mu$ s and may detect the rebound and the splash phenomena.

The speed of the camera was $2900 \mathrm{fps}$, with a pixel matrix of $64 \times 64$, i.e. a visual field of $2 \times 2 \mathrm{~mm}$, resulting in a local measurement. On each series a FFT was performed and three frequency peaks were found: very low $(1-2 \mathrm{~Hz})$, mean (about $40 \mathrm{~Hz}$ ) and high (from 100 to $1300 \mathrm{~Hz}$ ). Among the physical quantities involved, it was calculated that the maximum relative error occurred for the cavitation index (about $2.6 \%$ ). The repeatability of the present experiments was verified by three different methods.

\section{Experimental results}

Interested readers are referred to Castellani $[15,16]$ for the comprehensive presentation of results. 


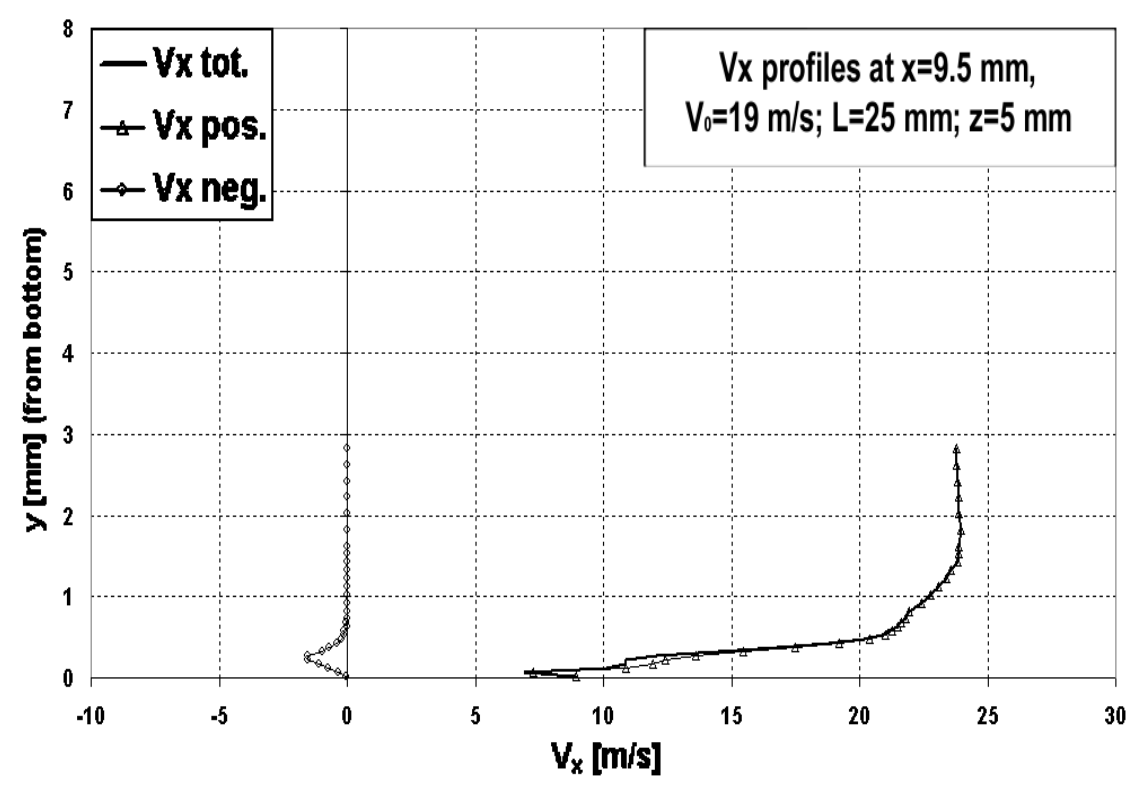

Figure 1: Negative $V_{x}$ for $L / c=0.56$ and $V_{0}=19 \mathrm{~m} / \mathrm{s}$.

The boundary layer detached at $x / c \simeq 0.6$ in any tested condition. It has also been numerically predicted. The experiments with cavitation were carried out at $L / c=0.34$ and $0.56, V_{0}=19,21$ and $29 \mathrm{~m} / \mathrm{s}$. Due to wall effects, it was not possible to produce a greater cavity length. The flow was chocked at $L / c \simeq 0.79$. The transitional cavitation was observed for $L / c \geq 0.5$; beyond this limit, surge cavitation appeared. The downstream turbulent transition is indicated by streaks on the cavity surface. The thickness of the cavity was deduced by differences between local and mean velocities and by the very high RMS of $V_{x}$.

At $x / c \simeq 0.5$, flow detachment is seen at the end of the cavity, where vapor is created and, for $L / c>0.5$, clouds. This is clear from fig. 1. Remarkably enough, this phenomenon occurs only at this location and does not depend on the cavity length $L / c$. For $x / c=0.5$ flow detachment is observed.

The profile shape seems to depend more on $V_{0}$ than on $\sigma_{0}$, suggesting that $\sigma_{0}$ alone may not wholly describe the phenomenon. The potential flow hypothesis has been verified by comparing the local measured velocity at the top border of the cavity with the theoretical one (Bernoulli's theorem).

A negative $V_{x}$ have been measured for $L / c=0.56$, starting from $x / c=0.21$ : a clear evidence of the re-entrant jet (fig. 1). The negative $V_{x}$ profile is maximum at $\sim 0.3 \mathrm{~mm}$ from the hydrofoil. This does not happen in other downstream stations, possibly indicating the upward rising of the jet.

The experiments to relate $\sigma$ to $L$ were carried out at constant velocity $V_{0}$. The tested range was $\sigma_{0} \in[0.72 ; 0.88]$. It was not possible to decrease $\sigma_{0}$ below 0.72 
(corresponding roughly to $L / c=0.68$ ) because of choking. The surge instability produces great cavity variations for small variations in $\sigma_{0}$ and was always detected for $L / c>0.67$. The wall effect caused this instability to be active for $L / c$ less than the literature value 0.75 (Watanabe [11]).

Incipient $(i)$ and desinent $(d)$ cavitation were investigated at $V_{0}=17,19,21$, 24,26 and $28 \mathrm{~m} / \mathrm{s}$. The difference between $\sigma_{0 i}$ and $\sigma_{0 d}$ was strongly affected by the time elapsed to vary the parameters $\left(p_{0}, V_{0}, \ldots\right)$ : the slower the variation, the greater the difference. Generally speaking, the diagrams $\sigma_{0}-L / c$ show an almost linear decrease of $\sigma_{0}$ and also a possible hysteretic behaviour. However experimental errors do not allow a doubtless interpretation for that matter.

The wall effect played an important role: in fact the variations of $L, p_{0}$ and $V_{0}$ were not simultaneous. The decrease of $V_{0}$ follows an increase of $p_{0}$, which in turn causes a decrease of $L$, in a self-induced cycle.

The pressure coefficient $C_{p}$ was measured along the hydrofoil at $V_{0} \in[15 ; 28]$ $\mathrm{m} / \mathrm{s}$ and $L / c \in[0 ; 0.8]$. In ideal conditions, the minimum pressure during cavitation would be $p_{v}$ : whence $\sigma_{i}=-C_{p, \min }$. As a matter of fact, $p_{\min } \leq p_{v}$ typically, resulting in $\sigma_{i} \leq-C_{p, \min }$. This parameter is useful to predict the starting location of the cavity and represents a limit for $\sigma_{i}$. It becomes important to know the difference between $\sigma_{i}$ and $C_{p, \min }$ because it shows the difference between theoretical and experimental conditions.

The diagrams $C_{P 0}$ vs. $x / c$ show an initial flat region, roughly corresponding to the cavity attached to the wall; then $C_{p 0}$ increases. It appears that the pressure coefficient is useful to describe the fixed part of the cavity. The flat region is actually shorter than the visually observed (by stroboscopic light, 2200 RPM) cavity length and this indicates that the re-entrant jet travels upstream under the cavity so that the pressure gauges are exposed directly to pure jet water and not to the vapor-water mixture of the cavity. An important result is that the extension of the flat region does not depend on the mean flow velocity.

The greatest difference between cavitating and non-cavitating flow has been observed for $L / c>0.5$.

The difference $\sigma_{0}-\left(-C_{p}\right)$ was plotted vs. $x / c$. In the flat region, this value is about 0.1 and it indicates that it is always $p_{\min }>p_{v}$. An useful graphical compact representation is showed in fig. 2 which shows an upper limit for $C_{p}$ depending on cavity length and $x$-location.

Three distinct frequencies were detected by means of a new non-intrusive method based on the recording of the laser scattered light at cavity closure: a laser beam was focused on the cavity surface and the scattered (indirect) light was recorded from a direction normal to the beam. Another setting made the beam to incide directly on the camera. The tested velocities were in the range $[15 ; 29.1] \mathrm{m} / \mathrm{s}$, while $L / c=0.3,0.4$ and 0.5 . This phenomenon does not pertain to the bubble collapse, but to the local interface vibration (indirect beam configuration) and to the vapour clouds motion (direct beam configuration).

There was not a well defined peak at the highest frequencies, but rather a frequency band, sometime as large as $400 \mathrm{~Hz}$.

The time scale for the mean flow to travel the distance $L$ can be defined as 


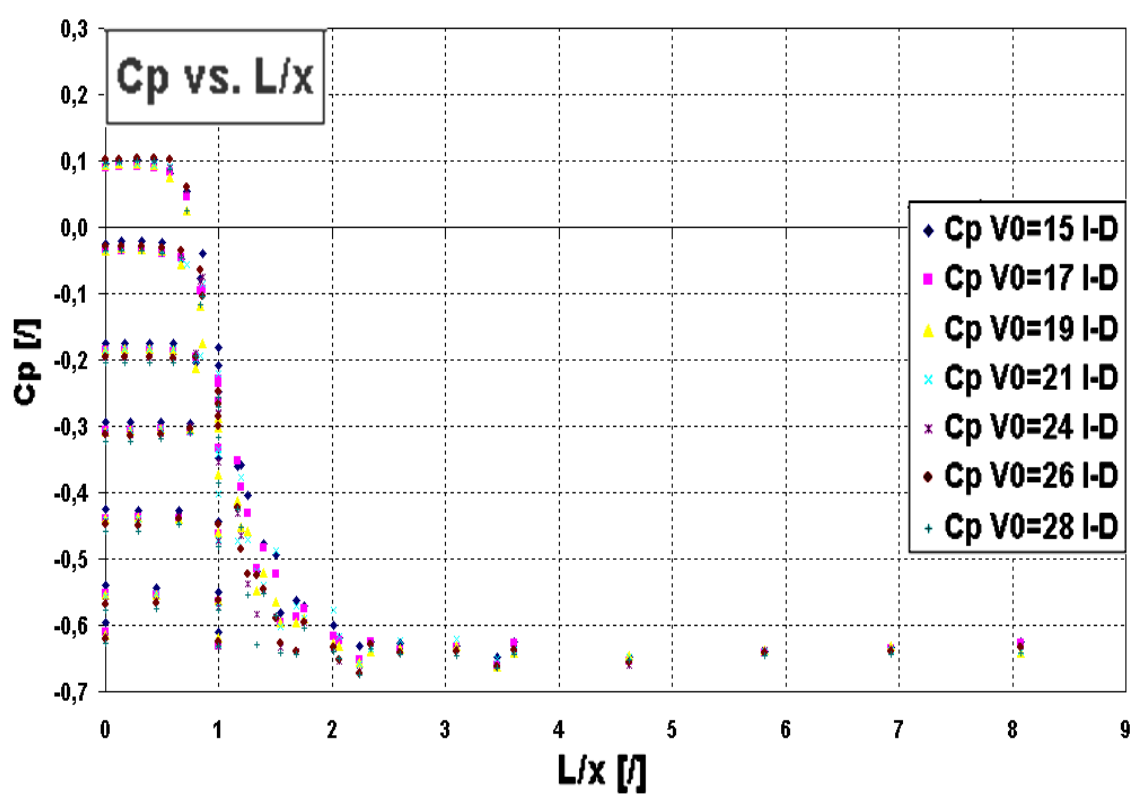

Figure 2: $C_{p}$ vs. $L / x$ for different velocities.

$\tau:=L / V$, whereas $1 / f$ is the time scale for the cavity to travel the distance $L$ twice (down- and upstream). The proposed Strouhal number is herein defined with $2 L$ as the length scale:

$$
S t:=\frac{2 f L}{V}=\frac{f}{1 / 2 \tau}
$$

The literature value for the vortex shedding behind a cylinder ranges around $S t \approx 0.3$.

The very low frequency peaks $(2-3 \mathrm{~Hz})$ are not typical of the cavity, and depend on the tunnel geometric configuration. Following Franc et al. [9], it can be defined a system instability. The same is true for the $40 \mathrm{~Hz}$ frequency, while the $100 \mathrm{~Hz}$ or greater frequencies seem quite specific.

The frequency content does not appear to depend upon $L / c$, but only on $V_{0}$. fig. 3 shows this dependence for St related to the peak and to the starting (min) and ending ( $\max$ ) frequency of each peak. St is always decreasing with $\sigma_{0}$. The highest frequency band may possibly indicate a chaotic interaction of different phenomena, where the spectrum is partly the sum and partly the convolution. Peaks were even observed at $200-300 \mathrm{~Hz}$ and at $1200-1300 \mathrm{~Hz}$. According to Kjeldsen [10], this is supposed to be the range governed by the re-entrant jet $\left(\sigma_{0} / 2 \alpha>4\right)$. St attains values up to 4 , an information not reported in the literature.

The instantaneous cavity length and thickness have also been measured. It is a global measure. The minimum recorded length is clearly increasing with $V_{0}$, fig. 4, 


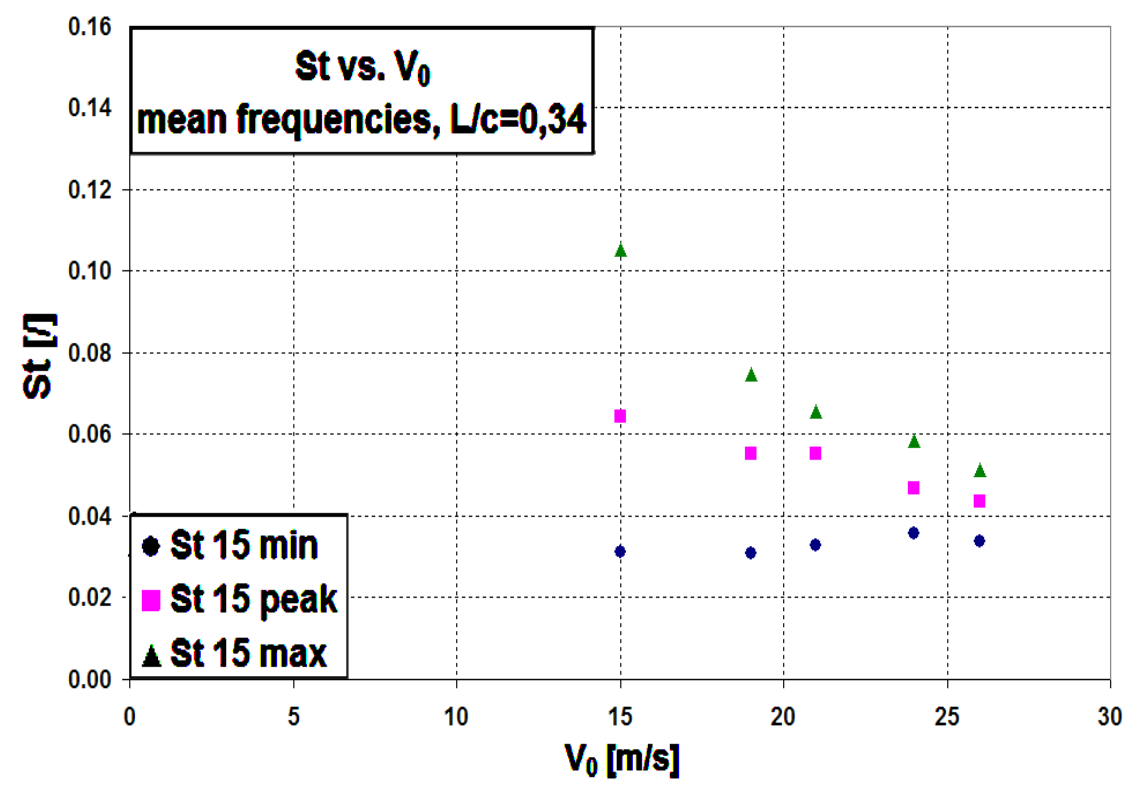

Figure 3: Strouhal number trend with velocity.

indicating that the length of the fixed part of the cavity is proportional to the length of the cavity. This fact may prevent the re-entrant jet to reach the upstream end of the cavity for long cavities. This is probably due to the low pressure gradient because of the wall effect. There are differences between this speculation and the results of Franc et al. [4, 5] and Kawanami et al. [6].

This result is confirmed also by the percentage of zero instantaneous thicknesses recorded for different velocities and lengths: for low velocities the cavity disappears, while this is not true at higher velocities.

$S t$ referred to $L_{c}$ is increasing with $V_{0}$ and $L / c$. If we consider the fixed part of the cavity, given by the minimum of $L_{c}$, we obtain an increasing trend with $V_{0}$ for all of the cavity lengths. This leads to an important consideration. If, according to Kawanami [6], $L_{c}$ is the cavity length, $L_{c u t}$ is the fixed cavity length and, following Franc [5], $\beta:=V_{j} / V_{0}=0.84$, where $V_{j}$ is the velocity of the re-entrant jet, it may be deduced that:

$$
\frac{f L_{c}}{V}=\frac{f L_{c u t}}{V}+\beta \frac{T_{j}}{T_{c}} \rightarrow \frac{T_{j}}{T_{c}}=\frac{L_{c}-L_{c u t}}{\beta V}
$$

If $V=V_{c}$, where $V_{c}:=\sqrt{1+\sigma_{0}}$ (Bernoulli), then:

$$
\frac{T_{j}}{T_{c}}=\frac{L_{c}-L_{c u t}}{0.84 V_{0} \sqrt{1+\sigma_{0}}}
$$




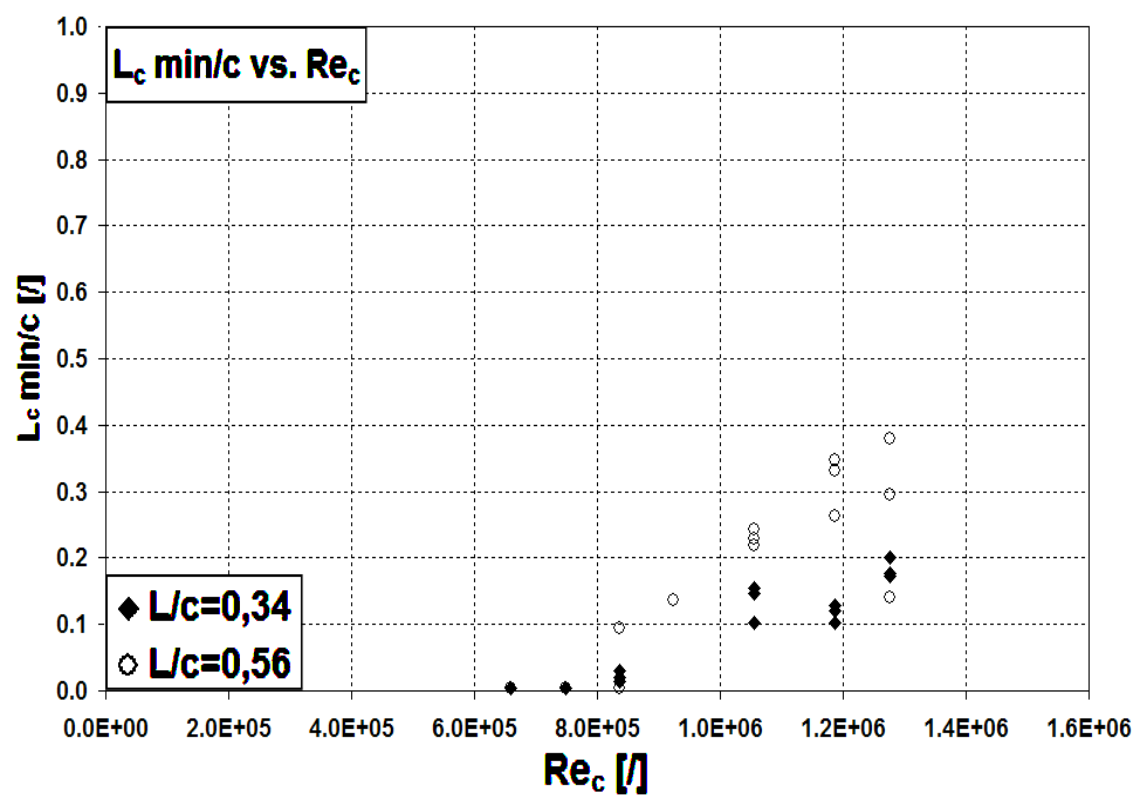

Figure 4: Minimum recorded cavity length for different $R e$.

Since the ratio $T_{j} / T_{c}$ decreases when $V_{0}$ increases, the time needed for the reentrant jet to travel upstream, compared with the time of cavity oscillation $\left(f^{-1}\right)$, decreases. This is reasonable because that ratio is acting as a Strouhal number of the mobile part of the cavity, scaled to $\beta$. The travel time of the re-entrant jet increases less than the corresponding increase of the cavity break time, so that the jet breaks shorter parts of the cavity. That means also that the cavity rebuilding velocity increases less than the velocity of the re-entrant jet.

As a result, the role of the re-entrant jet in breaking the cavity is not as much important as it is commonly thought. No correlation between frequency peaks and flow velocity have been detected.

As for the instantaneous pressure peaks, the experiments were performed at $V_{0} \in[18.5 ; 29] \mathrm{m} / \mathrm{s}$ and $L / c=0.56$ and the measurements were taken at $x / c \equiv$ $L / c$. Not only a decrease of $\sigma_{0}$ but also an increment of $V_{0}$ can produce surge instability, because of wall effects.

Analyzing directly the shape of the time series, fig. 5, it can be noticed (for low velocity, i.e. 18-24 m/s) some "valley", 10-20 ms wide, with high peaks inside. Outside the valley there were generally no peaks. These valleys represent the transit of the cavity border, or vapour clouds, while the peaks represent the pressure pulses given by bubbles implosions. Actually, the peak has a basis $\sim 1 \mathrm{~ms}$ wide in time and shows typically four damped oscillations. Moreover each sub-peak of this oscillation has often two spires, at an interval of some $0.05 \mathrm{~ms}$. These results are in agreement with the literature: the damped oscillation is related to the rebound, 


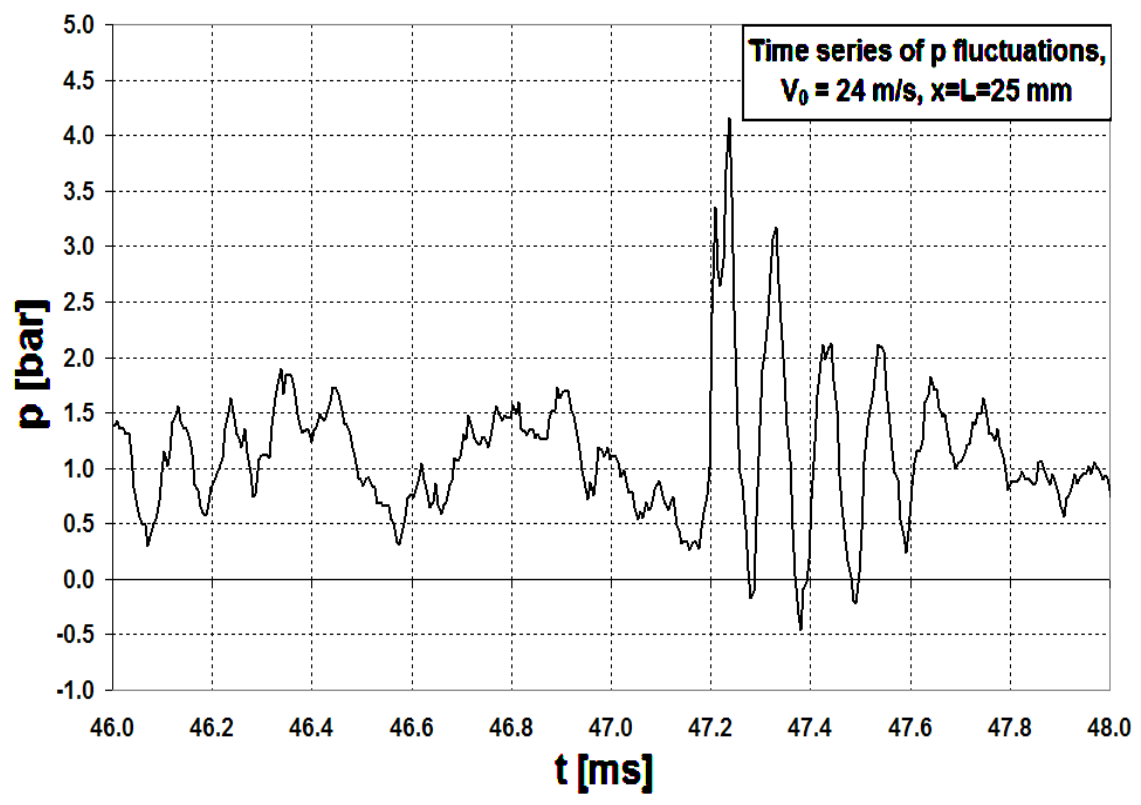

Figure 5: Visualization of rebound and splash phenomena.

and the double spire can be interpreted as the splash phenomenon, observed in Tong et al. [7].

The following parameters have been calculated:

$$
I 1:=\frac{1}{t} \sum_{i=1}^{N} n_{i} a_{i}
$$

which is an index of the cavitation intensity, and

$$
A 1:=\frac{\sum_{i=1}^{N} n_{i} a_{i}}{\sum_{i=1}^{N} n_{i}}
$$

which is an index of the average width of the peak, where $n_{i}$ is the peaks number in the $i$-class and $a_{i}$ is the $i$-class width. These parameters are both clearly increasing with $V_{0}$ and this indicates that, for the same $\sigma_{0}$, the cavity is stronger and more dangerous for higher velocities.

\section{Conclusions}

Sheet cavitation was observed, with a surge behaviour for high $L / c$, transitional behaviour for $L / c=0.56$ and choke for $L / c=0.79$. A great influence of the downstream pressure gradient was observed and $x / c \simeq 0.5$ was seen to be a threshold between two cavitation types (sheet-cloud) and a point of flow separation. The 
velocity profile was found to depend more upon $V_{0}$ than upon $\sigma_{0}$, so that the cavitation number cannot be exhaustive. The slowness of the parameter variation was found to dramatically affect the incipient cavitation. The wall effect was found to have a great importance. The cavitation number increases slowly with $V_{0}$ and only for $V_{0}>25 \mathrm{~m} / \mathrm{s}$. A limit point was found again for $L / c=0.55$. The observed negative velocities indicates the presence of the re-entrant jet. The extension of the constant $C_{p 0}$ region (attached and fixed cavity) was found not to be influenced by the velocity. The cavity actual pressure $p_{c}$ was always greater than $p_{v}$.

The frequency content of cavitation was spread on three regions: very low (2-3 $\mathrm{Hz}$ ), low (about $40 \mathrm{~Hz}$ ), possibly related to a system instability, and high (up to $1300 \mathrm{~Hz}$ ), which produces Strouhal numbers greater than those detected in the literature. A new method of cavity vibration measurement is proposed that is based on recording the scattered laser light at the cavity interface. The velocity of cavity rebuilding is not wholly controlled by the re-entrant jet, as eqn. (2) shows, suggesting that the role of the re-entrant jet in breaking the cavity should be partly reconsidered.

Three different methods of frequency detection have been compared and found in agreement. The Strouhal number for the highest frequencies of pressure pulses agrees with literature data (see Chandrashekhar and Syamala Rao [1]).

\section{References}

[1] Chandrashekhar, D.V., Syamala Rao, B.C., Effect of pressure on the length of cavity and cavitation damage behind circular cylinders in a venturi, Journal of Fluid Engineering, June 1973.

[2] Fry, S.A., The damage capacity of cavitating flow from pulse height analysis, Journal of Fluid Engineering, Vol. 102, December 1989.

[3] Ramamurthy, A.S., Balachandar, R., A note on choking cavitation flow past bluff bodies, Journal of Fluid Engineering, Vol. 114, September 1992.

[4] Franc, J.P., Michel, J.M., Le, Q., Partial cavities: pressure pulse distribution around cavity closure, Journal of Fluid Engineering, Vol. 115, June 1993.

[5] Franc, J.P., Michel, J.M., Le Q., Partial cavities: global behavior and mean pressure distribution, Journal of Fluid Engineering, Vol. 115, June 1993.

[6] Kawanami, Y., Kato, H., Yamaguchi, H., Three-dimensional characteristics of the cavities formed on a two-dimensional hydrofoil, Third International Symposium on Cavitation, Grenoble, 1998.

[7] Tong, R.P., Schiffers, W.P., Shaw, S.J., Blake, J.R., Emmony, D.C., The role of "splashing" in the collapse of a laser-generated cavity near a rigid boundary, Journal of Fluid Mechanics, Vol. 380, 1999.

[8] Astolfi, J.A., Dorange, P., Billard, J.Y., Cid, T.I., An experimental investigation of cavitation inception and development on a two dimensional Eppler hydrofoil, Journal of Fluid Engineering, Vol. 122, March 2000.

[9] Franc, J.P., Partial cavity instabilities and re-entrant jet, Fourth International Symposium on Cavitation, Pasadena, USA, 2001.

[10] Kjeldsen, M., Arndt, R.E.A., Joint time frequency analysis techniques: a 
study of transitional dynamics in sheet-cloud cavitation, Fourth International Symposium on Cavitation, Pasadena, USA, 2001.

[11] Watanabe, S., Tsusjimoto, Y., Furukawa, A., Theoretical analysis of transitional and partial cavity instabilities, Journal of Fluid Engineering, Vol. 123, September 2001.

[12] Lindau, O., Lauterborn, W., Cinematographic observation of the collapse and rebound of a laser-produced cavitation bubble near a wall, Journal of Fluid Mechanics, Vol. 479, 2002.

[13] Saito, Y., Sato, K., Cavitation bubble collapse and impact in the wake of a circular cylinder, Fifth International Symposium on Cavitation, Osaka, Japan, 2003.

[14] Sato, K., Shimojo, S., Detailed observations on a starting mechanism for shedding of cavitation cloud, Fifth International Symposium on Cavitation, Osaka, Japan, 2003.

[15] Castellani, I., Hydrodynamic 2D cavitation - experimental database, Mechanical and Structural Engineering Department, University of Trento, Laboratory report, Trento, 2005.

[16] Castellani, I., Studio di un profilo cavitante in un ugello venturi [Study of a cavitating hydrofoil in a Venturi channel], Ph.D thesis, Udine, 2005. 